\title{
Projective measurements and generation of entangled Dirac particles in Schwarzschild Spacetime
}

\author{
Jieci Wang, Qiyuan Pan and Jiliang Jing* \\ Institute of Physics and Department of Physics, \\ Hunan Normal University, Changsha, \\ Hunan 410081, P. R. China \\ and \\ Key Laboratory of Low-dimensional Quantum Structures \\ and Quantum Control of Ministry of Education, \\ Hunan Normal University, Changsha, \\ Hunan 410081, P. R. China
}

\begin{abstract}
It is shown that the projective measurements made by Bob who locates near the event horizon of the Schwarzschild black hole will create entangled particles detected by Alice who stays stationary at the asymptotically flat region. It is found that the degree of entanglement decreases as the frequency of the detected particles increases and approaches to zero as the frequency $\omega_{\mathbf{k}} \rightarrow \infty$. It is also noted that the degree of entanglement increases as the Hawking temperature increases. Especially, the particle state is unentangled when the Hawking temperature is zero and approaches a maximally entangled Bell state when the black hole evaporates completely.
\end{abstract}

PACS numbers: 03.65.Ud, 03.67.Mn, 04.70.Dy, 97.60.Lf

\footnotetext{
* Corresponding author, Email: jljing@hunnu.edu.cn
} 


\section{INTRODUCTION}

Quantum entanglement lies at the heart of quantum information theory, with applications to quantum computing, teleportation, and communication. It is widely accepted that understanding the entanglement in a relativistic framework is not only of interest to the quantum information, but also plays an important role in the black hole physics [1, 2]. Thus, much attention has been focus on the study of the quantum information in a relativistic setting [3 15]. Recently, it was found that, due to the Unruh effect [16], the projective measurements made by the accelerated observer can generate real particles detectable by the inertial observer both in the cases of the scalar [17] and Dirac [18] fields. Furthermore, as shown in [18], the entanglement of the produced state increase as the observer's acceleration increase.

As a further step along this line, the aim of this paper is to investigate how the projective measurements made by the observer who locates near the event horizon of the Schwarzschild black hole affect the Dirac particle state detected by observer who stays stationary at the asymptotically flat region and how the Hawking temperature [19] change the properties of the entanglement. Our scheme can be set up by two observers, Alice and Bob, together with their associated detectors. After the coincidence of Alice and Bob at the same initial point in an asymptotically flat region, Alice stays stationary at the flat place, while Bob falls toward the Schwarzschild black hole and hovers at a fixed finite nearest distance away from the horizon with uniform acceleration. As a result of the Hawking effect, Bob will perceive a Fermi-Dirac distribution of particles and antiparticles in the Kruskal vacuum. Then we let Bob make a standard von Neumann projective measurement (measuring the particle number) [17, 18] and figure out what Alice obtains. It is worth mentioning that if we let Bob always free fall, the projective measurement made by him also have an affect on Alices state. However, this is in fact another issue because Bob could not observes the existence of the event horizon.

The outline of the paper is as follows. In Sec. 2 we discuss the features of quantum field theory in the Schwarzschild spacetime and the Hawking effect for the Dirac particles. In Sec. 3 we analyze the effects of the projective measurements on the generation of entangled fermions in the Schwarzschild spacetime. We will summarize and discuss our conclusions in the last section. 


\section{VACUUM STRUCTURE AND HAWKING RADIATION FOR DIRAC FIELDS}

The metric for the Schwarzschild spacetime is given by

$$
d s^{2}=-\left(1-\frac{2 M}{r}\right) d t^{2}+\left(1-\frac{2 M}{r}\right)^{-1} d r^{2}+r^{2}\left(d \theta^{2}+\sin ^{2} \theta d \varphi^{2}\right),
$$

where the parameter $M$ represents the mass of the black hole. Throughout this paper we use $G=c=\hbar=\kappa_{B}=1$. Introducing a tortoise coordinate

$$
r_{*}=r+2 M \ln \frac{r-2 M}{2 M}
$$

and defining the advanced time $v$ and retarded time $u$ as

$$
v=t+r_{*} \quad u=t-r_{*},
$$

we obtain the generalized light-like Kruskal coordinates $\mathcal{U}$ and $\mathcal{V}$ for the Schwarzschild black hole [23]

$$
\begin{aligned}
& u=-4 M \ln \left(-\frac{\mathcal{U}}{4 M}\right), \quad v=4 M \ln \left(\frac{\mathcal{V}}{4 M}\right), \quad \text { if } \quad \mathrm{r}>\mathrm{r}_{+} \\
& u=-4 M \ln \left(\frac{\mathcal{U}}{4 M}\right), \quad v=4 M \ln \left(\frac{\mathcal{V}}{4 M}\right), \quad \text { if } \quad \mathrm{r}<\mathrm{r}_{+}
\end{aligned}
$$

where $\mathcal{U}$ and $\mathcal{V}$ are regular across the past and future horizons of the extended spacetime.

For the Schwarzschild spacetime the Dirac equation [20]

$$
\left[\gamma^{a} e_{a}{ }^{\mu}\left(\partial_{\mu}+\Gamma_{\mu}\right)\right] \Psi=0,
$$

can be written as

$$
\begin{array}{cc}
-\frac{\gamma_{0}}{\sqrt{1-\frac{2 M}{r}} \frac{\partial \Psi}{\partial t}}+\gamma_{1} \sqrt{1-\frac{2 M}{r}}\left[\frac{\partial}{\partial r}+\frac{1}{r}+\frac{M}{2 r(r-2 M)}\right] \Psi \\
+\frac{\gamma_{2}}{r}\left(\frac{\partial}{\partial \theta}+\frac{1}{2} \cot \theta\right) \Psi+\frac{\gamma_{3}}{r \sin \theta} \frac{\partial \Psi}{\partial \varphi}=0 .
\end{array}
$$

If we re-scale $\Psi$ as

$$
\Psi=\left(1-\frac{2 M}{r}\right)^{-\frac{1}{4}} \Phi,
$$

and use an ansatz for the Dirac spinor

$$
\Phi=\left(\begin{array}{c}
\frac{i \chi_{1}^{( \pm)}(r)}{r} \phi_{j m}^{ \pm}(\theta, \varphi) \\
\frac{\chi_{2}^{( \pm)}(r)}{r} \phi_{j m}^{\mp}(\theta, \varphi)
\end{array}\right) e^{-i \omega t}
$$


with spinor angular harmonics

$$
\begin{aligned}
& \phi_{j m}^{+}=\left(\begin{array}{c}
\sqrt{\frac{j+m}{2 j}} Y_{l}^{m-1 / 2} \\
\sqrt{\frac{j-m}{2 j}} Y_{l}^{m+1 / 2}
\end{array}\right), \quad\left(\text { for } j=l+\frac{1}{2}\right), \\
& \phi_{j m}^{-}=\left(\begin{array}{c}
\sqrt{\frac{j+1-m}{2 j+2}} Y_{l}^{m-1 / 2} \\
-\sqrt{\frac{j+1+m}{2 j+2}} Y_{l}^{m+1 / 2}
\end{array}\right), \quad\left(\text { for } j=l-\frac{1}{2}\right),
\end{aligned}
$$

we find that the cases for $(+)$ and $(-)$ in the functions $\chi_{1}^{( \pm)}$and $\chi_{2}^{( \pm)}$can be put together, and then the decoupled equations can be expressed as

$$
\begin{aligned}
& \frac{d^{2} \chi_{1}}{d r_{*}^{2}}+\left(\omega^{2}-V_{1}\right) \chi_{1}=0 \\
& \frac{d^{2} \chi_{2}}{d r_{*}^{2}}+\left(\omega^{2}-V_{2}\right) \chi_{2}=0
\end{aligned}
$$

with

$$
\begin{aligned}
& V_{1}=\frac{\sqrt{1-\frac{2 M}{r}}|K|}{r^{2}}\left(|K| \sqrt{1-\frac{2 M}{r}}+\frac{r-3 M}{r}\right), \quad\left(K=-j-\frac{1}{2}, \quad j=l-\frac{1}{2}\right), \\
& V_{2}=\frac{\sqrt{1-\frac{2 M}{r}}|K|}{r^{2}}\left(|K| \sqrt{1-\frac{2 M}{r}}-\frac{r-3 M}{r}\right), \quad\left(K=j+\frac{1}{2}, \quad j=l+\frac{1}{2}\right) .
\end{aligned}
$$

Solving Eqs. (12) and (13) near the event horizon, we obtain $\chi_{1}=\chi_{2}=e^{ \pm i \omega r_{*}}$. Hereafter we will use the wavevector $\mathbf{k}$ labels the modes. Particles and antiparticles will be classified with respect to the future-directed timelike Killing vector in each region [10]. Thus, for the outside and inside region of the event horizon, the positive (fermions) frequency outgoing solutions are found to be [23]

$$
\begin{aligned}
& \Psi_{\mathbf{k}}^{I+}=\mathcal{G} e^{-i \omega u}, \\
& \Psi_{\mathbf{k}}^{I I+}=\mathcal{G} e^{i \omega u},
\end{aligned}
$$

where

$$
\mathcal{G}=\left[\begin{array}{c}
i\left(r^{4}-2 M r^{3}\right)^{-\frac{1}{4}} \phi_{j m}^{ \pm}(\theta, \varphi) \\
\left(r^{4}-2 M r^{3}\right)^{-\frac{1}{4}} \phi_{j m}^{\mp}(\theta, \varphi)
\end{array}\right]
$$

is a 4-component Dirac spinor. 
Since the modes $\Psi_{\mathbf{k}}^{I+}$ and $\Psi_{\mathbf{k}}^{I+}$ are analytic outside and inside the event horizon respectively, they form a complete orthogonal family. Thus, in terms of these bases the field $\Psi_{\text {out }}$ can be expanded as

$$
\Psi_{\text {out }}=\sum_{\sigma} \int d \mathbf{k}\left[a_{\mathbf{k}}^{\sigma} \Psi_{\mathbf{k}}^{\sigma+}+b_{\mathbf{k}}^{\sigma \dagger} \Psi_{\mathbf{k}}^{\sigma-}\right]
$$

where $\sigma=(I, I I), a_{\mathbf{k}}^{I}$ and $b_{\mathbf{k}}^{I \dagger}$ are the fermion annihilation and antifermion creation operators acting on the state of the exterior region, and $a_{\mathbf{k}}^{I I}$ and $b_{\mathbf{k}}^{I I \dagger}$ are the fermion annihilation and antifermion creation operators acting on the state of the interior region of the Schwarzschild black hole, respectively.

On the other hand, by making an analytic continuation for Eqs. (16) and (17), we find a complete basis for positive energy modes which analytic for all real $\mathcal{U}$ and $\mathcal{V}$ according to the suggestion of Domour-Ruffini [23]

$$
\begin{gathered}
\mathscr{F}_{\mathbf{k}}^{I+}=e^{2 \pi M \omega_{\mathbf{k}}} \Psi_{\mathbf{k}}^{I+}+e^{-2 \pi M \omega_{\mathbf{k}}} \Psi_{-\mathbf{k}}^{I I-}, \\
\mathscr{F}_{\mathbf{k}}^{I I+}=e^{-2 \pi M \omega_{\mathbf{k}}} \Psi_{-\mathbf{k}}^{I-}+e^{2 \pi M \omega_{\mathbf{k}}} \Psi_{\mathbf{k}}^{I I+} .
\end{gathered}
$$

Thus, we can also quantize the Dirac field in the Kruskal spacetime as

$$
\Psi_{\text {out }}=\sum_{\sigma} \int d \mathbf{k}\left[2 \cosh \left(4 \pi M \omega_{\mathbf{k}}\right)\right]^{-1 / 2}\left[c_{\mathbf{k}}^{\sigma} \mathscr{F}_{\mathbf{k}}^{\sigma+}+d_{\mathbf{k}}^{\sigma \dagger} \mathscr{F}_{\mathbf{k}}^{\sigma-}\right],
$$

where $c_{\mathbf{k}}^{\sigma}$ and $d_{\mathbf{k}}^{\sigma \dagger}$ are the annihilation and creation operators acting on the Kruskal vacuum.

Eqs. (19) and (22) represent the decomposition of the Dirac field in Schwarzschild and Kruskal modes respectively, so we can easily get the Bogoliubov transformations [10]

$$
\left[\begin{array}{c}
c_{\mathbf{k}}^{\sigma} \\
d_{-\mathbf{k}}^{\sigma \dagger}
\end{array}\right]=V\left[\begin{array}{c}
a_{\mathbf{k}}^{\sigma} \\
b_{-\mathbf{k}}^{\sigma \dagger}
\end{array}\right] V^{-1}
$$

where $V=\exp \left[r\left(a_{\mathbf{k}}^{I \dagger} b_{-\mathbf{k}}^{I I \dagger}+a_{\mathbf{k}}^{I} b_{-\mathbf{k}}^{I I}\right)\right]$ is a two-mode Dirac squeezing operator.

Then we assume that the Kruskal vacuum $\left|0_{\mathbf{k}}\right\rangle_{\mathcal{K}}^{+}$is related to the vacuum of the black hole $\left|0_{\mathbf{k}}\right\rangle_{I}^{+}\left|0_{-\mathbf{k}}\right\rangle_{I I}^{-}$by

$$
\left|0_{\mathbf{k}}\right\rangle_{\mathcal{K}}^{+}=\digamma\left(a_{\mathbf{k}}^{I}, a_{\mathbf{k}}^{I \dagger}, b_{-\mathbf{k}}^{I I}, b_{-\mathbf{k}}^{I I \dagger}\right)\left|0_{\mathbf{k}}\right\rangle_{I}^{+}\left|0_{-\mathbf{k}}\right\rangle_{I I}^{-}
$$

From $\left[a_{\mathbf{k}}^{I}, a_{\mathbf{k}}^{I \dagger}\right]=\left[b_{-\mathbf{k}}^{I I}, b_{-\mathbf{k}}^{I I \dagger}\right]=1$ and $c_{\mathbf{k}}^{I}\left|0_{\mathbf{k}}\right\rangle_{\mathcal{K}}^{+}=0$, we get [13, 14, 16]

$$
\digamma \propto \exp \left(e^{-4 \pi M \omega_{\mathbf{k}}} a_{\mathbf{k}}^{I \dagger} b_{-\mathbf{k}}^{I I \dagger}\right)
$$


After properly normalizing the state vector, the Kruskal particle vacuum state for mode $\mathbf{k}$ is found to be

$$
\left|0_{\mathbf{k}}\right\rangle_{\mathcal{K}}^{+}=\left(e^{-8 \pi M \omega_{\mathbf{k}}}+1\right)^{-\frac{1}{2}} \exp \left(e^{-4 \pi M \omega_{\mathbf{k}}} a_{\mathbf{k}}^{I \dagger} b_{-\mathbf{k}}^{I I \dagger}\right)\left|0_{\mathbf{k}}\right\rangle_{I}^{+}\left|0_{-\mathbf{k}}\right\rangle_{I I}^{-},
$$

where $\left\{\left|n_{-\mathbf{k}}\right\rangle_{I I}^{-}\right\}$and $\left\{\left|n_{\mathbf{k}}\right\rangle_{I}^{+}\right\}$are the orthonormal bases for the inside and outside region of the event horizon respectively, and the $\{+,-\}$ superscript on the kets is used to indicate the particle and antiparticle vacua.

A formal expression for the total Kruskal particle vacuum is obtained by using Eq. (26) for each mode

$$
|0\rangle_{\mathcal{K}}^{+}=\prod_{\mathbf{k}}\left(e^{-8 \pi M \omega_{\mathbf{k}}}+1\right)^{-\frac{1}{2}} \exp \left(e^{-4 \pi M \omega_{\mathbf{k}}} a_{\mathbf{k}}^{I \dagger} b_{-\mathbf{k}}^{I I \dagger}\right)\left|0_{\mathbf{k}}\right\rangle_{I}^{+}\left|0_{-\mathbf{k}}\right\rangle_{I I}^{-} .
$$

Similarly, the total Kruskal antiparticle vacuum takes the form

$$
|0\rangle_{\mathcal{K}}^{-}=\prod_{\mathbf{k}}\left(e^{-8 \pi M \omega_{\mathbf{k}}}+1\right)^{-\frac{1}{2}} \exp \left(-e^{-4 \pi M \omega_{\mathbf{k}}} b_{\mathbf{k}}^{I \dagger} a_{-\mathbf{k}}^{I I \dagger}\right)\left|0_{\mathbf{k}}\right\rangle_{I}^{-}\left|0_{-\mathbf{k}}\right\rangle_{I I}^{+} .
$$

Then, the full Kruskal vacuum is $|0\rangle_{\mathcal{K}}=|0\rangle_{\mathcal{K}}^{+}|0\rangle_{\mathcal{K}}^{-}$, which corresponds to the absence of particles and antiparticles as detected by the Kruskal observer.

When Bob travels through the Kruskal vacuum, his detector registers the number of particles

$$
N_{\omega}^{2}=\frac{1}{e^{8 \pi M \omega}+1}=\frac{1}{e^{\omega / T}+1},
$$

which shows that the observer in the exterior of the black hole detects a thermal FermiDirac distribution of particles. Here we have defined the Hawking temperature as $T=\frac{1}{8 \pi M}$ $[25,26]$.

\section{GENERATING ENTANGLED FERMIONS BY PROJECTIVE MEASURE- MENTS}

We now study the relationships between projective measurements and the generation of entangled particles in the background of the Schwarzschild black hole. The vacuum (27) could be rewritten as

$$
\begin{aligned}
|0\rangle_{\mathcal{K}}^{+}= & \left(e^{-\omega_{\mathbf{k}} / T}+1\right)^{-\frac{1}{2}} \exp \left(e^{-\frac{\omega_{\mathbf{k}}}{2 T}} a_{\mathbf{k}}^{I \dagger} b_{-\mathbf{k}}^{I I \dagger}\right) \\
& \prod_{\mathbf{k}^{\prime} \neq \mathbf{k}}\left(e^{-\omega_{\mathbf{k}^{\prime}} / T}+1\right)^{-\frac{1}{2}} \exp \left(e^{-\frac{\omega_{\mathbf{k}^{\prime}}}{2 T}} a_{\mathbf{k}^{\prime}}^{I \dagger} b_{-\mathbf{k}^{\prime}}^{I I \dagger}\right)\left|0_{\mathbf{k}^{\prime}}\right\rangle_{I}^{+}\left|0_{-\mathbf{k}^{\prime}}\right\rangle_{I I}^{-}
\end{aligned}
$$


Now supposing that Bob performs a measurement on this state and detects one particle in the mode $\mathbf{k}$, then the state will be projected to the single particle state

$$
\left|\Phi_{+}(\mathbf{k})\right\rangle_{B}=\mathcal{P}_{\mathbf{k}} \prod_{\mathbf{k}^{\prime}}\left(e^{-\omega_{\mathbf{k}^{\prime}} / T}+1\right)^{-\frac{1}{2}} \exp \left(e^{-\frac{\omega_{\mathbf{k}^{\prime}}}{2 T}} a_{\mathbf{k}^{\prime}}^{I \dagger} b_{-\mathbf{k}^{\prime}}^{I \dagger \dagger}\right)\left|0_{\mathbf{k}^{\prime}}\right\rangle_{I}^{+}\left|0_{-\mathbf{k}^{\prime}}\right\rangle_{I I}^{-}
$$

where $\mathcal{P}_{\mathbf{k}}$ is defined as

$$
\mathcal{P}_{\mathbf{k}}=\left(e^{-\omega_{\mathbf{k}} / T}+1\right)^{\frac{1}{2}} a_{\mathbf{k}}^{I \dagger} b_{-\mathbf{k}}^{I I \dagger} \exp \left(-e^{-\frac{\omega_{\mathbf{k}}}{2 T}} a_{\mathbf{k}}^{I \dagger} b_{-\mathbf{k}}^{I I \dagger}\right)
$$

Then we switch back to the Alice's frame to figure out what she obtains [17, 18]. After some calculations for the Bogoliubov transformation, we obtain

$$
\begin{aligned}
\left|\Phi_{+}(\mathbf{k})\right\rangle & =V^{-1}\left|\Phi_{+}(\mathbf{k})\right\rangle_{B} \\
& =\left(e^{\omega_{\mathbf{k}} / T}+1\right)^{-\frac{1}{2}}\left|\tilde{0}_{\mathbf{k}}\right\rangle^{+}\left|\tilde{0}_{-\mathbf{k}}\right\rangle^{-}+\left(e^{-\omega_{\mathbf{k}} / T}+1\right)^{-\frac{1}{2}}\left|\tilde{1}_{\mathbf{k}}\right\rangle^{+}\left|\tilde{1}_{-\mathbf{k}}\right\rangle^{-},
\end{aligned}
$$

where $\left|\tilde{1}_{\mathbf{k}}\right\rangle^{+}$and $\left|\tilde{1}_{-\mathbf{k}}\right\rangle^{-}$are single particle and antiparticle excitation in Alice's detector. We see that from Alice's perspective, the state is a superposition of the vacuum and pair production of fermions an antifermions at frequency $\omega_{\mathbf{k}}$. The physical mechanism of this process can be given as follows. As a result of the Hawking effect, Bob will perceive a Fermi-Dirac distribution of particles and antiparticles. Since only a thermal bath in the accelerated frame corresponds to vacuum obtains by Alice [17], a measurement by Bob will destroy the purely thermal nature of the field, then Alice can no longer obtain a vacuum state.

In the case of a supermassive or an almost extreme black hole $(T \rightarrow 0)$, the state $\left|\Phi_{+}(\mathbf{k})\right\rangle$ approaches to

$$
\lim _{T \rightarrow 0}\left|\Phi_{+}(\mathbf{k})\right\rangle=\left|\tilde{1}_{\mathbf{k}}\right\rangle^{+}\left|\tilde{1}_{-\mathbf{k}}\right\rangle^{-}
$$

It is interesting to note that the projective measurement made by Bob also created particles detectable by Alice in the case of $T \rightarrow 0$, but this particle state is unentangled in this case.

And in the limit $T \rightarrow \infty$, which corresponds to the case of the black hole evaporates completely, the state $\left|\Phi_{+}(\mathbf{k})\right\rangle$ approaches a maximally entangled Bell state

$$
\lim _{T \rightarrow \infty}\left|\Phi_{+}(\mathbf{k})\right\rangle=\frac{1}{\sqrt{2}}\left(\left|\tilde{0}_{\mathbf{k}}\right\rangle^{+}\left|\tilde{0}_{-\mathbf{k}}\right\rangle^{-}+\left|\tilde{1}_{\mathbf{k}}\right\rangle^{+}\left|\tilde{1}_{-\mathbf{k}}\right\rangle^{-}\right) .
$$

It is well known that the degree of bipartite entanglement can be quantified uniquely for pure states by the partial entropy of entanglement, defined as the von Neumann entropy 
[9, 27]

$$
E_{p}(|\psi\rangle)=S\left(\rho_{A}\right)=S\left(\rho_{B}\right)=-\sum_{i} \lambda_{i} \log _{2} \lambda_{i}
$$

where $\rho_{A}$ (or $\rho_{B}$ ) is the reduced density matrix of subsystem $A$ (or $B$ ), and $\lambda_{i}$ is the $i$ th eigenvalues of $\rho_{A}$ or $\rho_{B}$.

The state (32) can be represented by the density matrix

$$
\begin{aligned}
\rho_{\mathbf{k}}= & \left(e^{\omega_{\mathbf{k}} / T}+1\right)^{-1}|\tilde{0} \tilde{0}\rangle\left\langle\tilde{0} \tilde{0}\left|+\left(e^{\omega_{\mathbf{k}} / T}+e^{-\omega_{\mathbf{k}} / T}+2\right)^{-\frac{1}{2}}\right| \tilde{0} \tilde{0}\right\rangle\langle\tilde{1} \tilde{1}| \\
& +\left(e^{\omega_{\mathbf{k}} / T}+e^{-\omega_{\mathbf{k}} / T}+2\right)^{-\frac{1}{2}}|\tilde{1} \tilde{1}\rangle\left\langle\tilde{0} \tilde{0}\left|+\left(e^{-\omega_{\mathbf{k}} / T}+1\right)^{-1}\right| \tilde{1} \tilde{1}\right\rangle\langle\tilde{1} \tilde{1}|,
\end{aligned}
$$

where $|\tilde{n} \tilde{m}\rangle=\left|\tilde{n}_{\mathbf{k}}\right\rangle^{+} \otimes\left|\tilde{m}_{-\mathbf{k}}\right\rangle^{-}$. In order to find the partial entropy of the state, we trace out one subsystem of the density matrix and obtain

$$
\rho_{\mathbf{k}}^{+}=\operatorname{Tr}_{-}\left(\rho_{\mathbf{k}}\right)=\left(e^{\omega_{\mathbf{k}} / T}+1\right)^{-1}|\tilde{0}\rangle\left\langle\tilde{0}\left|+\left(e^{-\omega_{\mathbf{k}} / T}+1\right)^{-1}\right| \tilde{1}\right\rangle\langle\tilde{1}|,
$$

where $|\tilde{n}\rangle=\left|\tilde{n}_{\mathbf{k}}\right\rangle^{+}$. From which the partial entropy of entanglement is found to be

$$
E_{p}\left[\left|\Phi_{+}(\mathbf{k})\right\rangle\right]=\log _{2}\left(e^{\omega_{\mathbf{k}} / T}+1\right)+\left(e^{-\omega_{\mathbf{k}} / T}+1\right)^{-1} \log _{2}\left(e^{-\omega_{\mathbf{k}} / T}\right) .
$$

The behaviors of the partial entropy of entanglement as a function of the frequency of the detected particles and the Hawking temperature $T$ are illustrated Fig.1, We find that, in the case of a supermassive or an almost extreme black hole $(T \rightarrow 0)$, the degree of entanglement is zero, which verifies that the state is an uncorrelated pure state. The state always entangled for nonzero Hawking temperature, and higher Hawking temperature producing more entanglement. As $T \rightarrow \infty$, corresponding to the case of the black hole evaporates completely, the degree of entanglement is $E_{p}\left[\left|\Phi_{+}(\mathbf{k})\right\rangle\right]=1$, which just verifies that the state in this case is the maximally entangled Bell state.

If Bob detects an antiparticle in mode $\mathbf{k}$, the resulting state can be simplifies to

$$
\left|\Phi_{-}(\mathbf{k})\right\rangle=\left[\left(e^{\omega_{\mathbf{k}} / T}+1\right)^{-\frac{1}{2}}-\left(e^{-\omega_{\mathbf{k}} / T}+1\right)^{-\frac{1}{2}} d_{\mathbf{k}}^{I \dagger} c_{-\mathbf{k}}^{I I \dagger}\right]\left|\tilde{0}_{\mathbf{k}}\right\rangle^{-}\left|\tilde{0}_{-\mathbf{k}}\right\rangle^{+},
$$

which is entangled in the occupation number of the antiparticle mode $\mathbf{k}$ and the particle mode $\mathbf{- k}$. This state also approaches a new Bell state

$$
\lim _{T \rightarrow \infty}\left|\Phi_{-}(\mathbf{k})\right\rangle=\frac{1}{\sqrt{2}}\left(\left|\tilde{0}_{\mathbf{k}}\right\rangle^{-}\left|\tilde{0}_{-\mathbf{k}}\right\rangle^{+}+\left|\tilde{1}_{\mathbf{k}}\right\rangle^{-}\left|\tilde{1}_{-\mathbf{k}}\right\rangle^{+}\right),
$$

when the black hole evaporates completely. And it approaches the uncorrelated pure state

$$
\lim _{T \rightarrow 0}\left|\Phi_{-}(\mathbf{k})\right\rangle=\left|\tilde{1}_{\mathbf{k}}\right\rangle^{-}\left|\tilde{1}_{-\mathbf{k}}\right\rangle^{+}
$$

in the case of supermassive or an almost extreme black hole. 


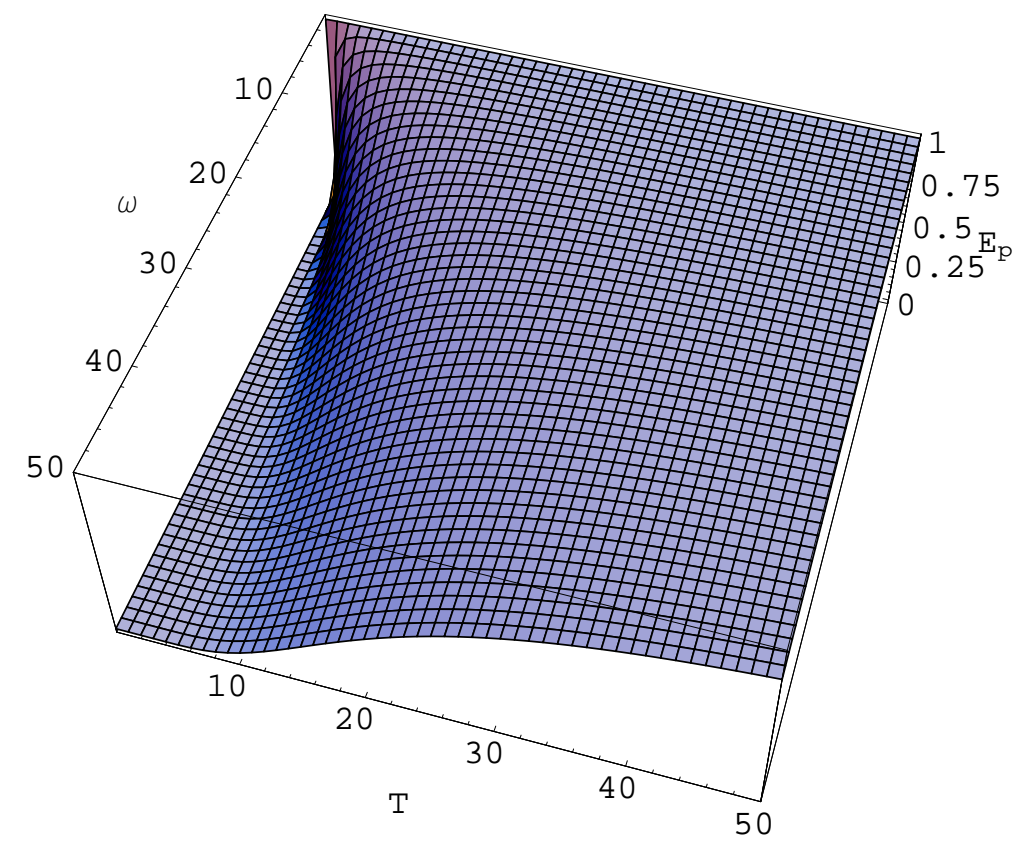

FIG. 1: The degree of entanglement as a function of the frequency of the detected particles $\omega_{\mathbf{k}}$ and the Hawking temperature $T$. It demonstrates that only the lower frequency particles are highly entangled. As the frequency $\omega_{\mathbf{k}} \rightarrow \infty$, the degree of entanglement decreases to zero.

\section{SUMMARY}

Building on the well-known Hawking effect, we have discussed the effects of projective measurements on the generation of entangled particles between two Dirac modes. It is shown that the projective measurements made by Bob who falls toward a Schwarzschild black hole will create entangled particles detected by Alice who stays stationary at the asymptotically flat region. The physical mechanism of this process is that once Bob makes a measurement, he destroys the purely thermal nature of the field, then Alice no longer obtains vacuum. We have demonstrated that only the lower frequency particles are highly entangled. As the frequency $\omega_{\mathbf{k}} \rightarrow \infty$, the degree of entanglement decreases to zero, that is to say, the state is an unentangled pure state. It is interesting to note that, when the Hawking temperature is zero, i.e., the case of supermassive or an almost extreme black hole, the projective measurement also created particles detectable by Alice, but the state is unentangled in this case. For nonzero Hawking temperature, the state is always entangled 
and its degree of entanglement increases as the Hawking temperature increases. In the limit of infinite Hawking temperature, i.e., the black hole evaporates completely, the degree of entanglement is exactly one, which just indicates that a maximally entangled Bell state is produced.

\section{Acknowledgments}

This work was supported by the National Natural Science Foundation of China under Grant No 10875040; the key project of the National Natural Science Foundation of China under Grant No 10935013; the National Basic Research of China under Grant No. 2010CB833004, the Hunan Provincial Natural Science Foundation of China under Grant No. 08JJ3010, PCSIRT, No. IRT0964, and Construct Program of the National Key Discipline.

[1] L. Bombelli, R. K. Koul, J. Lee, and R. Sorkin, Phys. Rev. D 34, 373 (1986).

[2] S. W. Hawking, Commun. Math. Phys. 43, 199 (1975); Phys. Rev. D 14, 2460 (1976); H. Terashima, Phys. Rev. D 61, 104016 (2000).

[3] A. Peres and D. R. Terno, Rev. Mod. Phys. 76, 93 (2004).

[4] D. Boschi, S. Branca, F. De Martini, L. Hardy, and S. Popescu, Phys. Rev. Lett. 80, 1121 (1998).

[5] D. Bouwmeester, A. Ekert, and A. Zeilinger, The Physics of Quantum Information (SpringerVerlag, Berlin), 2000.

[6] P. M. Alsing and G. J. Milburn, Phys. Rev. Lett. 91, 180404 (2003).

[7] P. M. Alsing, D. McMahon, and G. J. Milburn, J. Opt. B: Quantum Semiclassical Opt. 6, S834 (2004).

[8] Xian-Hui Ge and Sang Pyo Kim, Class. Quantum Grav. 25, 075011 (2008).

[9] I. Fuentes-Schuller and R. B. Mann, Phys. Rev. Lett. 95, 120404 (2005).

[10] P. M. Alsing, I. Fuentes-Schuller, R. B. Mann, and T. E. Tessier, Phys. Rev. A 74, 032326 (2006).

[11] G. Adesso, I. Fuentes-Schuller, and M. Ericsson, Phys. Rev. A 76, 062112 (2007).

[12] Qiyuan Pan and Jiliang Jing, Phys. Rev. A 77, 024302 (2008). 
[13] Qiyuan Pan and Jiliang Jing, Phys. Rev. D 78, 065015 (2008).

[14] D. Ahn, Y. H. Moon, R. B. Mann, and I. Fuentes-Schuller, JHEP 0806, 062 (2008).

[15] Jason Doukas and Lloyd C. L. Hollenberg Phys. Rev. A 79, 052109 (2009)

[16] W. G. Unruh, Phys. Rev. D 14, 870 (1976).

[17] M. Han, J. S. Olson, and J. P. Dowling, Phys. Rev. A 78, 022302 (2008).

[18] David C. M. Ostapchuk and Robert B. Mann, Phys. Rev. A 79, 042333 (2009).

[19] S. W. Hawking, Nature 248, 30 (1974).

[20] D. R. Brill and J. A. Wheeler, Rev. Mod. Phys. 29, 465 (1957); 45, 3888(E) (1992).

[21] Jiliang Jing, Phys. Rev. D 70, 065004 (2004).

[22] Jiliang Jing, Phys. Rev. D 69, 084009 (2004).

[23] T. Damoar and R. Ruffini, Phys. Rev. D 14, 332 (1976).

[24] S. M. Barnett and P. M. Radmore, Methods in Theoretical Quantum Optics, 67-80 (Oxford University Press, New York), 1997.

[25] R. Kerner and R. B. Mann, Phys. Rev. D 73, 104010 (2006).

[26] Qing-Quan Jiang, Shuang-Qing Wu, and Xu Cai, Phys. Rev. D 75, 064029 (2007); ShuangQing Wu, ibid. 76, 029904(E) (2007).

[27] M. A. Nielsen and I. L. Chuang, Quantum Computation and Quantum Information (Cambridge University Press, Cambridge), 2000. 